УДК 544.[344+228]:546.[683+48+28+289+81]'23/24

DOI https://doi.org/10.32782/pcsd-2021-2-5

\title{
Іван ОЛЕКСЕЮК
}

доктор хімічних наук, професор, професор кафедри хімії та технологій Волинський національний університет імені Лесі Украӥнки, просп. Волі, 13, м. Луцьк, Волинська обл., Украӥна, 43025

ORCID: 0000-0001-7206-4351

\section{Андрій СЕЛЕЗЕНЬ}

аспірант, Волинський національний університет імені Лесі Українки, просп. Волі, 13, м. Луцьк, Волинська обл., Украӥна, 43025

ORCID: 0000-0002-1174-7439

\section{Олександр СМІТЮХ}

кандидат хімічних наук, старший лаборант кафедри хімії та технологій, Волинський національний університет імені Лесі Українки, просп. Волі, 13, м. Луиьк, Волинська обл., Україна, 43025

ORCID: 0000-0003-1632-5849

\section{Любомир ГУЛАЙ}

доктор хімічних наук, професор, завідувач кафедри екології та природокористування, Волинський національний університет імені Лесі Українки, просп. Волі, 13, м. Луцьк, Волинська обл., Україна, 43025 ORCID: 0000-0003-3495-5027

\section{Людмила ПІСКАЧ}

кандидат хімічних наук, професор, професор кафедри хімії та технологій, Волинський наиіональний університет імені Лесі Украӥнки, просп. Волі, 13, м. Луиьк, Волинська обл., Україна, 43025

ORCID: 0000-0003-3117-4006

Scopus AuthorID: 6603765788.

Бібліографічний опис статті: Олексеюк, I., Селезень, А., Смітюх, О., Гулай, Л., Піскач, Л. (2021). Тетрарні халькогеніди систем $\mathrm{Tl}_{2} \mathrm{X}-\mathrm{B}^{\mathrm{II}} \mathrm{X}-\mathrm{D}^{\mathrm{IV}} \mathrm{X}_{2}$ ( $\left.\mathrm{B}^{\mathrm{II}}-\mathrm{Cd}, \mathrm{Hg}, \mathrm{D}^{\mathrm{IV}}-\mathrm{Si}, \mathrm{Ge} ; \mathrm{X}-\mathrm{Se}, \mathrm{Te}\right)$. Проблеми хімії та сталого розвитку, 2, 26-37, doi: https://doi.org/10.32782/pcsd-2021-2-5

\section{ТЕТРАРНІ ХАЛЬКОГЕНІДИ СИСТЕМ ТІ ${ }_{2} \mathrm{X}-\mathrm{B}^{\mathrm{II}} \mathrm{X}-\mathrm{D}^{\mathrm{IV}} \mathrm{X}_{2}\left(\mathrm{~B}^{\mathrm{II}}-\mathrm{Cd}, \mathrm{Hg}, \mathrm{D}^{\mathrm{IV}}-\mathrm{Si}, \mathrm{Ge} ; \mathrm{X}-\mathrm{Se}, \mathrm{Te}\right)$}

за результатами ренгенофазового аналізу побудовано ізотермічні перерізи систем $\mathrm{Tl}_{2} \mathrm{Se}-\mathrm{CdSe}-\mathrm{Ge}(\mathrm{Sn}) \mathrm{Se} \mathrm{e}_{2}$ при $570 \mathrm{~K}$.

У системі $\mathrm{Tl}_{2} \mathrm{Se}-\mathrm{CdSe-GeSe} \mathrm{S}_{2}$ за температури відпалу у стані термодинамічної рівноваги встановлено утво-

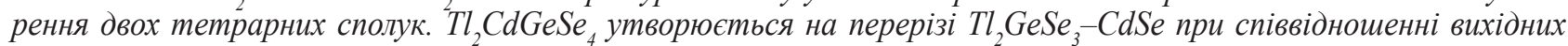

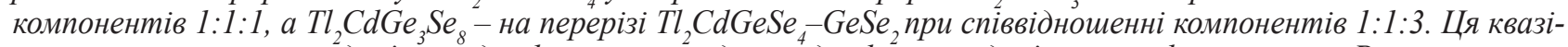
потрійна система має дев'ять однофазних, сімнадиять двофазних і дев'ять трифазних полів. Розчинність на основі CdSe по перерізах $\mathrm{Tl}_{4} \mathrm{GeSe}_{4}-\mathrm{CdSe} \mathrm{ma} \mathrm{Tl}_{2} \mathrm{GeSe}_{3}-\mathrm{CdSe}$ знаходиться в межах 3 мол.\%.

B системі $\mathrm{Tl}_{2} \mathrm{Se}-\mathrm{CdSe}-\mathrm{SnSe}{ }_{2}$ nри $570 \mathrm{~K}$ підтверджено існування сполуки $\mathrm{Tl}_{2} \mathrm{CdSnSe}{ }_{4}$ та зафіксовано наявність шести однофазних, десяти двофазних і п'яти трифазних полів.

Розшифровано кристалічну структуру чотирьох тетрарних сполук: $\mathrm{Tl}_{2} \mathrm{CdGe} \mathrm{Se}_{8}$ та трьох ізоструктурних халькогенідів: $\mathrm{Tl}_{2} \mathrm{CdGeSe}, \mathrm{Tl}_{2} \mathrm{CdSiTe}, \mathrm{Tl}_{2} \mathrm{HgSiTe}_{4}$. $\mathrm{Tl}_{2} \mathrm{CdGe} \mathrm{Se}_{8}$ кристалізується в тригональній ПГ P2 $22_{1} 2_{1} 3$ параметрами: $a=0.7 .6023(9), b=1.2071(2), c=1.7474(2)$ нм. $\mathrm{Tl}_{2} B^{I I} D^{I V} X_{4}$ кристалізуються в тетрагональній структурі з ПГ I-42m. Параметри комірок сполук: $a=0.80145(9), c=0.67234(9)$ нм (Tl, CdGeSe $) ; a=0.8049(6), c$ $=0.68573(8) \mathrm{HM}\left(\mathrm{Tl}_{2} \mathrm{CdSnSe}\right.$ ); $a=0.84121(6), c=0.70289(9) \mathrm{HM}\left(\mathrm{Tl}_{2} \mathrm{CdSiTe}_{4}\right) ; a=0.83929(4), \mathrm{c}=0.70396(5) \mathrm{HM}$ ( $\mathrm{Tl}_{2} \mathrm{HgSiTe}$ ). Розглянуто залежність об'єму просторової гратки та розрахованої густини від молярної маси у одинадияти відомих раніше та трьох нововиявлених ізоструктурних (ПГ I-42m) сполуках $T_{2} B^{I I} D^{I V} X_{4}$. Pозглянуто залежність об'єму просторової гратки та розрахованої густини від молярної маси у одинадияти відомих раніме та трьох нововиявлених ізоструктурних (ПГ I-42m) сполуках $T_{2} B^{I I} D^{I V} X_{4}$.

Ключові слова: талієвмісні тетрарні халькогеніди, фазові рівноваги, кристалічна структура, ренттенофазовий аналіз. 


\section{Ivan OLEKSEYUK}

Doctor of Chemical Sciences, Professor, Professor at the Department of Chemistry and Technology, Lesya Ukrainka Volyn National University, 13 Voli ave., Lutsk, Volyn region, Ukraine, 43025

ORCID: 0000-0001-7206-4351

\section{Andrii SELEZEN}

Postgraduate, Lesya Ukrainka Volyn National University, 13 Voli ave., Lutsk, Volyn region, Ukraine, 43025 ORCID: 0000-0002-1174-7439

\section{Oleksandr SMITIUKH}

PhD in Chemistry, Senior Assistant at the Department of Chemistry and Technology, Lesya Ukrainka Volyn National University, 13 Voli ave., Lutsk, Volyn region, Ukraine, 43025

ORCID: 0000-0003-1632-5849

\section{Liubomyr HULAI}

Doctor of Chemistry, Professor, Head of the Department of Ecology and Nature Management, Lesya Ukrainka Volyn National University, 13 Voli ave., Lutsk, Volyn region, Ukraine, 43025

ORCID: 0000-0003-3495-5027

\section{Lyudmyla PISKACH}

PhD in Chemistry, Professor, Professor at the Department of Chemistry and Technology, Lesya Ukrainka Volyn National University, 13 Voli ave., Lutsk, Volyn region, Ukraine, 43025

ORCID: 0000-0003-3117-4006

To cite this article: Olekseyuk, I., Selezen, A., Smitiukh, O., Hulai, L. \& Piskach, L. (2021). Tetrarni khalkohenidy system $\mathrm{Tl}_{2} \mathrm{X}-\mathrm{B}^{\mathrm{II}} \mathrm{X}-\mathrm{D}^{\mathrm{IV}} \mathrm{X}_{2}\left(\mathrm{~B}^{\mathrm{II}}-\mathrm{Cd}, \mathrm{Hg}, \mathrm{D}^{\mathrm{IV}}-\mathrm{Si}, \mathrm{Ge} ; \mathrm{X}-\mathrm{Se}, \mathrm{Te}\right)$. [Four-elements chalcogenides of the systems $\mathrm{Tl}_{2} \mathrm{X}-\mathrm{B}^{\mathrm{II}} \mathrm{X}-\mathrm{D}^{\mathrm{IV}} \mathrm{X}_{2}\left(\mathrm{~B}^{\mathrm{II}}-\mathrm{Cd}, \mathrm{Hg}, \mathrm{D}^{\mathrm{IV}}-\mathrm{Si}, \mathrm{Ge} ; \mathrm{X}-\mathrm{Se}, \mathrm{Te}\right)$. Problems of Chemistry and Sustainable Development, 2, 26-37, doi: https://doi.org/10.32782/pcsd-2021-2-5

\section{FOUR-ELEMENTS CHALCOGENIDES OF THE SYSTEMS $\mathrm{Tl}_{2} \mathrm{X}-\mathrm{B}^{\mathrm{II}} \mathrm{X}-\mathrm{D}^{\mathrm{IV}} \mathrm{X}_{2}\left(\mathrm{~B}^{\mathrm{II}}-\mathrm{Cd}, \mathrm{Hg}, \mathrm{D}^{\mathrm{IV}}-\mathrm{Si}, \mathrm{Ge}\right.$; $\left.\mathrm{X}-\mathrm{Se}, \mathrm{Te}\right)$}

Isothermal sections of the $\mathrm{Tl}, \mathrm{Se}-\mathrm{CdSe}-\mathrm{Ge}(\mathrm{Sn}) \mathrm{Se}$, systems at $570 \mathrm{~K}$ were investigated by XRD results. The formation of the two quaternary compounds was found in the $\mathrm{Tl}_{2} \mathrm{Se}-\mathrm{CdSe}-\mathrm{GeSe} e_{2}$ system at the annealing temperature in the state of thermodynamic equilibrium. $\mathrm{Tl}_{2} \mathrm{CdGeSe} \mathrm{e}_{4}$ forms at the $\mathrm{Tl}_{2} \mathrm{GeSe} \mathrm{e}_{3} \mathrm{CdSe}$ section at 1:1:1 ratio of the initial components,

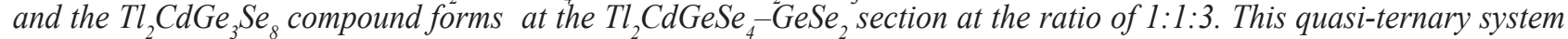
contains nine single-phase, seventeen two-phase, and nine three-phase fields. The solid solubility range of CdSe is within 3 mol.\% at the $\mathrm{Tl}_{4} \mathrm{GeSe}_{4}-\mathrm{CdSe}$ and $\mathrm{Tl}_{2} \mathrm{GeSe}_{3}-\mathrm{CdSe}$ sections.

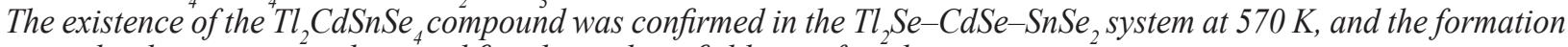
of six single-phase, ten two-phase and five three-phase fields was found.

The crystal structure of four quaternary compounds, $\mathrm{Tl}_{2} \mathrm{CdGe} \mathrm{Se}_{8}$ and three isostructural chalcogenides $\mathrm{Tl}_{2} \mathrm{CdGeSe_{4 }}$ $\mathrm{Tl}_{2} \mathrm{CdSiTe} \mathrm{Tl}_{4} \mathrm{Tl}_{2} \mathrm{SiTe}$, was determined. $\mathrm{Tl}_{2} \mathrm{CdGe} \mathrm{Se}_{8}$ crystallizes in the orthorhombic symmetry, $\mathrm{SG} P 2_{1} 2_{1}{ }_{1}$, lattice parameters $a=0.76023(9), b=1.2071(2), c=1.7474(2) \mathrm{nm} . T l_{2} B^{I I} D^{I V} X_{4}$ crystallizes in the tetragonal structure, SG $I-42 \mathrm{~m}$. The cell parameters of the compounds are: $a=0.80145(9), c=0.67234(9) \mathrm{nm}\left(\mathrm{Tl}_{2} \mathrm{CdGeSe}\right) ; a=0.8049(6), c=0.68573(8)$ $\mathrm{nm}\left(\mathrm{Tl}_{2} \mathrm{CdSnSe}\right.$ ); $a=0.84121(6), c=0.70289(9) \mathrm{nm}$ (Tl, CdSiTe $) ; a=0.83929(4), c=0.70396(5) \mathrm{nm}\left(\mathrm{Tl}_{2} \mathrm{HgSiTe}_{4}\right) . \mathrm{The}_{4}$ dependence of the unit cell volume and the calculated density on the molar mass in eleven previously known and three newly discovered isostructural (SG I-42m) $\mathrm{Tl}_{2} B^{I I} D^{I V} X_{4}$ compounds was considered.

Key words: thallium-containing quaternary chalcogenides, phase equilibria, crystal structure, X-ray phase analysis.

Дослідження халькогенідних систем $\mathrm{A}^{\mathrm{I}-\mathrm{B}^{\mathrm{II}}-}$ $\mathrm{D}^{\mathrm{IV}}-\mathrm{X}\left(\mathrm{B}^{\mathrm{II}}-\mathrm{Mn}, \mathrm{Fe}, \mathrm{Co}, \mathrm{Ni}, \mathrm{Cd}, \mathrm{Hg}, \mathrm{Pb} ; \mathrm{D}^{\mathrm{IV}}-\right.$ $\mathrm{Si}, \mathrm{Ge}, \mathrm{Sn} ; \mathrm{X}-\mathrm{S}, \mathrm{Se}, \mathrm{Te})$ за участю $\mathrm{A}^{\mathrm{I}}$ - лужних металів, $\mathrm{Cu}, \mathrm{Ag}$ значно розширює область пошуку нових напівпровідникових матеріалів.
У таких системах на квазіпотрійних перерізах $\mathrm{A}_{2}^{\mathrm{I}} \mathrm{X}-\mathrm{B}^{\mathrm{II}} \mathrm{X}-\mathrm{D}^{\mathrm{IV}} \mathrm{X}_{2}$ утворюються тетрарні фази типу $\mathrm{A}_{2}^{\mathrm{I}} \mathrm{B}^{\mathrm{II}} \mathrm{D}^{\mathrm{IV}} \mathrm{X}_{4}[1-10]$, окремі представники яких, уже зарекомендували себе в нелінійній оптиці та інших напрямках напівпровідникових 
технологій. Зокрема, такі тетрарні сполуки як $\mathrm{Li}_{2} \mathrm{ZnGeS}_{4}, \mathrm{Li}_{2} \mathrm{CdGeSe}_{4}, \mathrm{Li}_{2} \mathrm{CdSnSe}_{4}, \mathrm{Cu}_{2} \mathrm{CdSiS}_{4}$, $\mathrm{Cu}_{2} \mathrm{CdGeSe}_{4}, \mathrm{Cu}_{2} \mathrm{CdSnSe}_{4}, \mathrm{Cu}_{2} \mathrm{ZnSiS}_{4}, \mathrm{Ag}_{2} \mathrm{ZnSnS}_{4}$, що належать до алмазоподібних напівпровідників із нормальною валентністю, проявляють високу ефективність генерації другої гармоніки, мають високу теплову стабільність та інші оптичні та термоелектричні властивості, через що $є$ перспективними в застосуванні як елементи сонячних батареях та інших електронних пристроїв [11-19].

Іони $\mathrm{Tl}^{+1}$ подібні до іонів лужних металів. Слід чекати, що атоми лужних металів у алмазоподібних фазах можна замінити атомами Талію і отримати ізоструктурні сполуки. Перші дослідження тетрарних сполук систем Tl-B ${ }^{\text {II }}$ $\mathrm{D}^{\mathrm{IV}}-\mathrm{X}$ розпочаті у $80-\mathrm{x}$ роках $\mathrm{XX}$ ст., через що кількість робіт є обмеженою. Зокрема, при сплавлянні стехіометричних кількостей талій(I), плюмбум(II) та германій(IV) сульфідів отримано тетрарну сполуку $\mathrm{Tl}_{2} \mathrm{PbGeS}_{4}$, яка кристалізується в нецентросиметричній моноклінній структурі (ПГ $\left.P 2_{1} / a\right)$ [20]. $\mathrm{У}$ роботі [21] наведені результати вивчення кристалічної структури ряду тетрарних телуридів $\left(\mathrm{Tl}_{2} \mathrm{MnGeTe}_{4}, \mathrm{Tl}_{2} \mathrm{MnSnTe}_{4}, \mathrm{Tl}_{2} \mathrm{CdGeTe}_{4}\right.$, $\mathrm{Tl}_{2} \mathrm{CdSnTe}_{4}, \mathrm{Tl}_{2} \mathrm{HgGeTe}_{4}, \mathrm{Tl}_{2} \mathrm{HgSnTe}_{4}$ ). Ці сполуки ізоструктурні та кристалізуються в тетрагональній ПГ I-42m. Під час дослідження сульфуро- та селеновмісних квазіпотрійних систем $\mathrm{Tl}_{2} \mathrm{X}-\mathrm{HgX}-\mathrm{D}^{\mathrm{IV}} \mathrm{X}_{2}$ були виявлені сполуки типу $\mathrm{Tl}_{2} \mathrm{HgD}^{\mathrm{IV}} \mathrm{X}_{4}$ [22-24]. Структуру чотирьох із них ( $\mathrm{Tl}_{2} \mathrm{HgSiSe}_{4}[22], \mathrm{Tl}_{2} \mathrm{HgGeSe}_{4}$ [23], $\mathrm{Tl}_{2} \mathrm{HgSnS}_{4}$ [24], $\mathrm{Tl}_{2} \mathrm{HgSnSe}_{4}$ [22]) розшифровано в ізотропному наближенні в межах моделі структури сполуки $\mathrm{Tl}_{2} \mathrm{HgGeTe}_{4}$ [22]. Індексування дифрактограм показало належність їх кристалічної структури до ПГ $I-42 m$. Такою ж структурою володіє отримана нами на перерізі $\mathrm{Tl}_{2} \mathrm{SnSe}_{3}-\mathrm{CdSe}$ сполука $\mathrm{Tl}_{2} \mathrm{CdSnSe}_{4}$ [25]. Серед аналогічних талієвмісних тетрарних сполук, знайдених у системах $\mathrm{Tl}_{2} \mathrm{X}-\mathrm{PbX}-\mathrm{D}^{\mathrm{IV}} \mathrm{X}_{2}$, визначено кристалічну структуру для $\mathrm{Tl}_{2} \mathrm{PbSiS}_{4}$ (моноклінна, ПГ $P 2_{1} / a$ ) [26], яка $\epsilon$ ізоструктурною до $\mathrm{Tl}_{2} \mathrm{PbGeS}_{4}$. Для ряду сполук $\mathrm{Tl}_{2} \mathrm{~B}^{\mathrm{II}} \mathrm{D}^{\mathrm{IV}} \mathrm{Te}_{4}\left(\mathrm{~B}^{\mathrm{II}}-\mathrm{Mn}, \mathrm{Cd}, \mathrm{Hg} ; \mathrm{D}^{\mathrm{IV}}-\right.$ $\mathrm{Si}, \mathrm{Ge}, \mathrm{Sn})$ в роботі [21] досліджувалися термоелектричні властивості. Тетрарні талієвмісні сполуки, що характеризуються нецетросиметричною природою кристалічної структури, схильні до генерації другої гармоніки, двофотонної адсорбції, п’єзоелектричних ефектів та інших нелінійно-оптичних властивостей [27-31].

В роботі представляються результати дослідження фізико-хімічної взаємодії в системах $\mathrm{Tl}_{2} \mathrm{Se}-\mathrm{CdSe}-\mathrm{Ge}(\mathrm{Sn}) \mathrm{Se}_{2}$ та розшифрувааання кристалічної структури чотирьох нових тетрарних сполук.

Вихідні бінарні сполуки $\mathrm{Tl}_{2} \mathrm{Se}, \mathrm{CdSe}, \mathrm{GeSe}_{2}$, $\mathrm{SnSe}_{2}$ у досліджуваних квазіпотрійних системах плавляться конгруентно $\left(\mathrm{Tl}_{2} \mathrm{Se}\right.$ при 660 К, CdSe при 1509 K, GeSe 2 при $1015 \mathrm{~K}$ та $\mathrm{SnSe}_{2}$ при 948 К) і є дальтонідами $[32,33]$. У трьох обмежуючих системах $\mathrm{Tl}_{2} \mathrm{Se}-\mathrm{GeSe}_{2}$, $\mathrm{Tl}_{2} \mathrm{Se}-\mathrm{SnSe}_{2}$ та $\mathrm{CdSe}-\mathrm{GeSe}_{2}$ утворюються тетрарні сполуки $\mathrm{Tl}_{4} \mathrm{GeSe}_{4}, \mathrm{Tl}_{2} \mathrm{GeSe}_{3}, \mathrm{Tl}_{2} \mathrm{Ge}_{2} \mathrm{Se}_{5}$, $\mathrm{Tl}_{4} \mathrm{SnSe}_{4}, \mathrm{Tl}_{2} \mathrm{SnSe}_{3}, \mathrm{Cd}_{4} \mathrm{GeSe}_{6}$ [34-38]. Ці сполуки характеризуються змішаним іонно-ковалентним зв'язком з різним ступенем іонності. Їх кристалографічні характеристики опубліковано в [32, 33, 39-47] та наведено в табл. 1.

Синтез зразків здійснювали однотемпературним методом в муфельній печі МП-60 сплавлянням простих речовин: талію, кадмію, германію, олова, селену (телуру) (вміст основного компонента 99,999 мас. \%) та попередньо синтезованого $\mathrm{HgTe}(\mathrm{Hg} 99,999$ мас. \%) у вакуумованих до тиску $1 \times 10^{-2}$ мм.рт.ст. кварцових ампулах. Режим синтезу зразків наступний: нагрів до 950 К зі швидкістю 20 К/год, 5 год витримки; нагрів до 1200 К (сіліцієвмісних зразків - до 1450 К) зі швидкістю 10 К/год, 5 год витримки. Охолодження до 570 К зі швидкістю 10 К/год і гомогенізуючий відпал за цієї температури 350 год. Після цього зразки загартовували у $20 \%$-ий водний розчин $\mathrm{NaCl}$. Всього в системі $\mathrm{Tl}_{2} \mathrm{Se}-\mathrm{CdSe}-\mathrm{GeSe}_{2}$ синтезовано 62 зразки, у системі $\mathrm{Tl}_{2} \mathrm{Se}-\mathrm{CdSe}-\mathrm{SnSe}_{2}-57$ зразків.

Порошкові рентгенограми для встановлення фазового складу синтезованих зразків отримували на дифрактометрі DRON 4-13 при К $\alpha$-випромінюванні в діапазоні $10^{\circ} \leq 2 \theta \leq 80^{\circ}$. Кристалічну структуру нових тетрарних халькогенідів розраховували методом Рітвельда 3 використанням програмного пакету WinCSD [48]. Візуалізація елементів кристалічної структури проведена за допомогою програмного забезпечення Diamond.

За результатами рентгенофазового аналізу побудовано ізотермічні перерізи двох систем $\mathrm{Tl}_{2} \mathrm{Se}-\mathrm{CdSe}-\mathrm{Ge}(\mathrm{Sn}) \mathrm{Se}_{2}$ за $570 \mathrm{~K}$. Підтверджено утворення бінарних $\mathrm{Tl}_{2} \mathrm{Se}, \mathrm{CdSe}, \mathrm{GeSe}_{2}$, 
Таблиця 1

Кристалографічні характеристики бінарних та потрійних сполук у системах $\mathrm{Tl}_{2} \mathrm{Se}-\mathrm{CdSe}-\mathrm{Ge}(\mathrm{Sn}) \mathrm{Se}_{2}$

\begin{tabular}{|c|c|c|c|c|c|}
\hline \multirow{2}{*}{ Сполука } & \multirow{2}{*}{ ПГ } & \multicolumn{3}{|c|}{ Параметри гратки, им } & \multirow{2}{*}{ Л-ра } \\
\hline & & a & b & c & \\
\hline $\mathrm{Tl}_{2} \mathrm{Se}$ & $P 4 / n c c$ & 0.852 & - & 1.268 & {$[32,39]$} \\
\hline \multirow{2}{*}{$\mathrm{CdSe}$} & \multirow{2}{*}{$\begin{array}{l}P 6_{3} m c \\
F-43 m\end{array}$} & 0.4309 & - & 0.7021 & \multirow{2}{*}{ [32] } \\
\hline & & 0.6084 & - & - & \\
\hline $\mathrm{GeSe}_{2}$ & $P 2_{1} / c$ & 0.7016 & $\begin{array}{c}1.6796 \\
\beta=90.65^{\circ}\end{array}$ & 1.1831 & [40] \\
\hline $\mathrm{SnSe}_{2}$ & $P-3 m^{1}$ & 0.3811 & - & 0.6137 & [41] \\
\hline $\mathrm{Tl}_{4} \mathrm{GeSe}_{4}$ & $C 2 / c$ & 1.1670 & $\begin{array}{c}0.7317 \\
\beta=106.54^{\circ}\end{array}$ & 2.5603 & [42] \\
\hline $\mathrm{Tl}_{2} \mathrm{GeSe}_{3}$ & $P-1$ & $\begin{array}{c}0.6925 \\
\alpha=90.55^{\circ}\end{array}$ & $\begin{array}{c}0.6934 \\
\beta=111.42^{\circ}\end{array}$ & $\begin{array}{c}0.8771 \\
\gamma=114.45^{\circ}\end{array}$ & [43] \\
\hline $\mathrm{Tl}_{2} \mathrm{Ge}_{2} \mathrm{Se}_{5}$ & $C 2 / c$ & 1.5602 & $\begin{array}{c}1.5549 \\
\beta=107.10^{\circ}\end{array}$ & 0.9052 & [44] \\
\hline $\mathrm{Tl}_{4} \mathrm{SnSe}_{4}$ & $P 2_{1} / c$ & $0.8491(3)$ & $\begin{array}{c}0.8400(7) \\
\beta=102.39^{\circ}\end{array}$ & $1.580(1)$ & [45] \\
\hline $\mathrm{Tl}_{2} \mathrm{SnSe}_{3}$ & Pnam & 0.8051 & 0.8169 & 2.124 & {$[46]$} \\
\hline $\mathrm{Cd}_{4} \mathrm{GeSe}_{6}$ & $C c$ & 1.2842 & $\begin{array}{c}0.7405 \\
\beta=109.825^{\circ}\end{array}$ & 1.2850 & [47] \\
\hline
\end{tabular}

$\mathrm{SnSe}_{2}$ і тернарних сполук $\mathrm{Tl}_{4} \mathrm{SnSe}_{4}, \mathrm{Tl}_{2} \mathrm{SnSe}_{3}$, $\mathrm{Tl}_{4} \mathrm{GeSe}_{4}, \mathrm{Tl}_{2} \mathrm{GeSe}_{3}, \mathrm{Tl}_{2} \mathrm{Ge}_{2} \mathrm{Se}_{5}, \mathrm{Cd}_{4} \mathrm{GeSe}_{6}$ у відповідних системах. Результати ідентифікації цих сполук добре узгоджуються 3 літературними даними [32, 33, 39-47].

Ізотермічний переріз системи $\mathrm{Tl}_{2} \mathrm{Se}-\mathrm{CdSe}-$ $\mathrm{GeSe}_{2}$ за температури $570 \mathrm{~K}$ зображено на рис. 1. $\mathrm{Tl}_{2} \mathrm{CdGeSe}_{4}$ утворюється на перерізі $\mathrm{Tl}_{2} \mathrm{GeSe}_{3}-\mathrm{CdSe}$ при співвідношенні вихідних компонентів 1:1:1, а $\mathrm{Tl}_{2} \mathrm{CdGe}_{3} \mathrm{Se}_{8}-$ на перерізі $\mathrm{Tl}_{2} \mathrm{CdGeSe}_{4}-\mathrm{GeSe}_{2}$ при співвідношенні компонентів 1:1:3. В системі за температури відпалу у стані термодинамічної рівноваги перебуває дев'ять однофазних, сімнадцять двофазних і дев'ять трифазних полів. Розчинність на основі $\mathrm{CdSe}$ по перерізах $\mathrm{Tl}_{4} \mathrm{GeSe}_{4}-\mathrm{CdSe}$ та $\mathrm{Tl}_{2} \mathrm{GeSe}_{3}-\mathrm{CdSe}$ знаходиться в межах 3 мол. $\%$.

Ізотермічний переріз системи $\mathrm{Tl}_{2} \mathrm{Se}-\mathrm{CdSe}-\mathrm{SnSe}_{2}$ при $570 \mathrm{~K}$ представлено на рис. 2. В цій системі при 570 К наявні шість однофазних, десять двофазних і п’ять трифазних полів. Підтверждено утворення нової тетрарної фази $\mathrm{Tl}_{2} \mathrm{CdSnSe}_{4}$, що утворюється на квазібінарному перерізі $\mathrm{Tl}_{2} \mathrm{SnSe}_{3}-\mathrm{CdSe}$,якмиповідомлялираніше у [24]. Розчинність для $\mathrm{Tl}_{4} \mathrm{SnSe}_{4}$ складає 6 мол.\% $\mathrm{CdSe}$ вздовж перерізу
$\mathrm{Tl}_{4} \mathrm{SnSe}_{4}-\mathrm{CdSe}$,для $\mathrm{Tl}_{2} \mathrm{Se}-3$ мол.\%вздовжперерізу $\mathrm{Tl}_{2} \mathrm{Se}-\mathrm{CdSe}$, для $\mathrm{CdSe}-3$ мол.\% вздовж перерізів $\mathrm{Tl}_{2} \mathrm{Se}-\mathrm{CdSe}, \mathrm{Tl}_{4} \mathrm{SnSe}_{4}-\mathrm{CdSe}, \mathrm{Tl}_{2} \mathrm{SnSe}_{3}-\mathrm{CdSe}$.

Враховуючи утворення цих сполук, додатково було синтезовано два аналогічні тетрарні халькогеніди. Визначено кристалографічні параметри для $\mathrm{Tl}_{2} \mathrm{CdSiTe}{ }_{4} \mathrm{Ta} \mathrm{Tl}_{2} \mathrm{HgSiTe}_{4}$.

Нові тетрарні сполуки $\mathrm{Tl}_{2} \mathrm{CdGeSe}_{4}, \mathrm{Tl}_{2} \mathrm{CdSiTe}_{4}$, $\mathrm{Tl}_{2} \mathrm{HgSiTe}_{4} \in$ ізоструктурними та кристалізуються в нецентросиметричній тетрагональній сингонії ПГ I-42m, символ Пірсона $t I 16$.

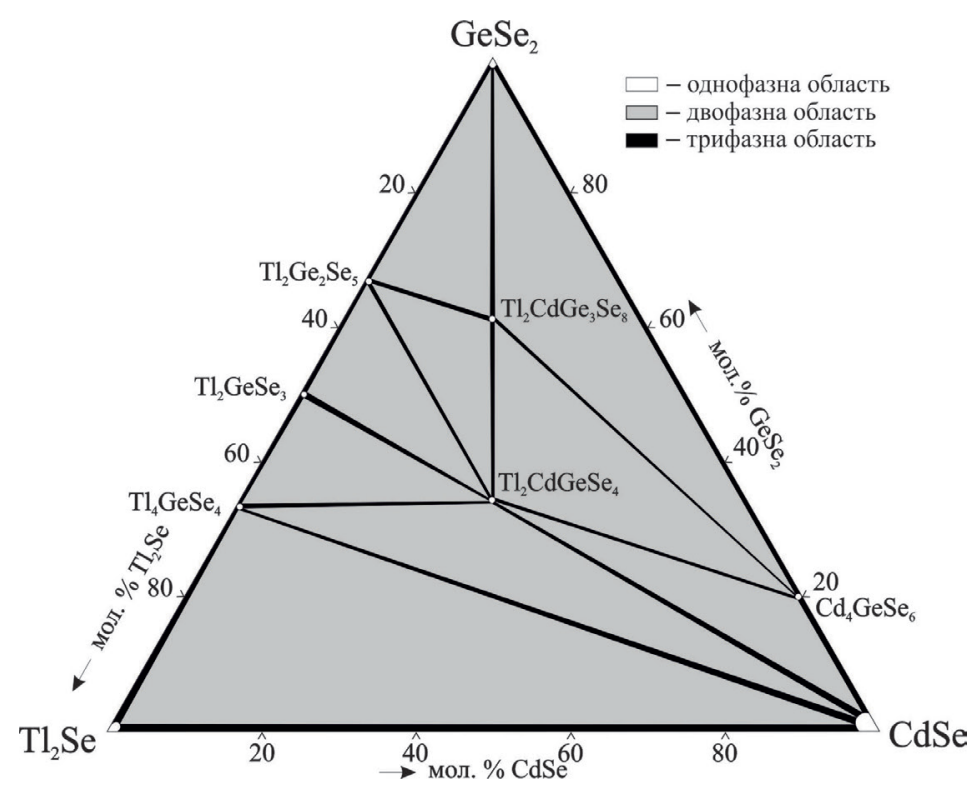

Рис. 1. Ізотермічний переріз квазіпотрійної системи $\mathrm{Tl}_{2} \mathrm{Se}-\mathrm{CdSe}-\mathrm{GeSe}_{2}$ при $570 \mathrm{~K}$ 


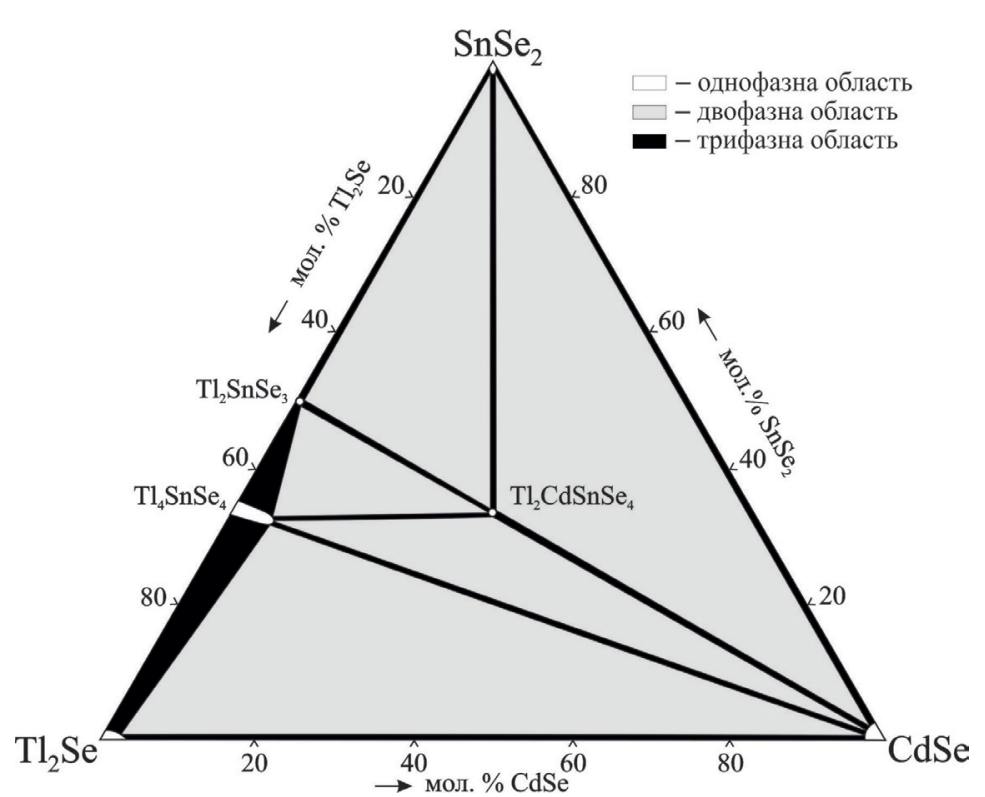

Рис. 2. Ізотермічний переріз квазіпотрійної системи $\mathrm{Tl}_{2} \mathrm{Se}-\mathrm{CdSe}-\mathrm{SnSe}_{2}$ при $570 \mathrm{~K}$

Ïх структуру розшифровано в ізотропному наближенні в межах моделі структури сполуки $\mathrm{Tl}_{2} \mathrm{HgGeTe}_{4}$ [20] та представлено у табл. 2 (дані для $\mathrm{Tl}_{2} \mathrm{CdSnSe}_{4}$ взяті з статті [24]). За розташуванням атомів халькогену в межах другого координаційного оточення (ДКО), структура досліджуваних сполук подібна до структури сполуки TlSe [48] - анізотропного напівпровідника з ланцюговою структурою складу $\mathrm{Tl}^{+}\left[\mathrm{Tl}^{3+} \mathrm{Se}_{2}\right]$, або до сполуки TIInSe ${ }_{2}$ [49], що виступає надструктурою до неї, у яких атоми одно- та тривалентного Талію

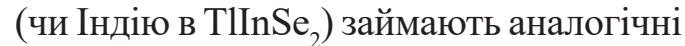
положення у вузлах катіонної підгратки і мають таку ж аніонну підгратку. Обидві сполуки кристалізуються в тетрагональній гратці з ПГ I4/mcm.

На рис. 3 наведені експериментальні, теоретичні та різницеві між ними рентгено-дифракційні спектри вищезазначених тетрарних сполук.

Параметри елементарних комірок сполук $\mathrm{Tl}_{2} \mathrm{~B}^{\mathrm{II}} \mathrm{D}^{\mathrm{IV}} \mathrm{X}_{4}$, які кристалізуються в тетрагональній сингонії ПГ $I-42 m$, в основному узгоджуються з загальновідомими закономірностями і перебувають в залежності від природи атомів. В більшості випадків при збільшенні порядкових номерів і відповідно маси атомів, що входять у склад сполуки, збільшуються густина і розміри атома. В табл. 3 представлено зміну об'єму елементарної комірки та густини відносно суми порядкових номерів елементів у сполуках $\mathrm{Tl}_{2} \mathrm{~B}^{\mathrm{II}} \mathrm{D}^{\mathrm{IV}} \mathrm{X}_{4}$.

Для порівняння були використані параметри гратки нових сполук та аналогічних талійвмісних тетрарних сполук 3 подібною будовою. Природа d-металів незначно впливає на розмірні параметри: заміна $\mathrm{Mn} \rightarrow \mathrm{Cd}$ у сполуках сприяе незначноному збільшенню, a $\mathrm{Cd} \rightarrow \mathrm{Hg}$ - незначному зменшенню кристалографічних параметрів комірки.

Таблиця 2

Результати розшифрування кристалічної структури сполук $\mathrm{Tl}_{2} \mathrm{~B}^{\mathrm{II}} \mathrm{D}^{\mathrm{IV}} \mathrm{X}_{4}$

\begin{tabular}{|c|c|c|c|c|}
\hline Емпірична формула & $\mathrm{Tl}_{2} \mathrm{CdGeSe}_{4}[24]$ & $\mathrm{Tl}_{2} \mathrm{CdSnSe}_{4}$ & $\mathrm{Tl}_{2} \mathrm{CdSiTe}_{4}$ & $\mathrm{Tl}_{2} \mathrm{HgSiTe}_{4}$ \\
\hline Просторова група & \multicolumn{4}{|c|}{$I-42 m$} \\
\hline Формульна маса & 909.57 & 955.67 & 1059.626 & 1147.84 \\
\hline \multicolumn{5}{|l|}{ Параметри комірки: } \\
\hline$a(\mathrm{HM})$ & $0.80145(9)$ & $0.80490(6)$ & $0.84121(6)$ & $0.83929(4)$ \\
\hline$c$ (нм) & $0.67234(9)$ & $0.68573(8)$ & $0.70289(9)$ & $0.70396(5)$ \\
\hline$V\left(\mathrm{HM}^{3}\right)$ & $0.4319(2)$ & $0.4443(1)$ & $0.4974(2)$ & $0.49587(9)$ \\
\hline $\mathrm{F}(000)$ & 756.0 & 792.0 & 864.0 & 928.0 \\
\hline Кількість атомних позицій & 16.0 & 16.0 & 16.0 & 16.0 \\
\hline 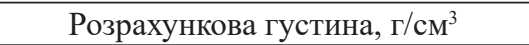 & $6.995(3)$ & $7.144(2)$ & $7.075(2)$ & $7.687(2)$ \\
\hline Коефіцієнт абсорбції, 1/см & 1167.94 & 1321.39 & 1756.94 & 1878.61 \\
\hline Випромінювання; довжина хвилі, нм & \multicolumn{4}{|c|}{$\mathrm{CuK}_{\sigma} \quad 0,154056$} \\
\hline Дифрактометр & \multicolumn{4}{|c|}{ Порошковий } \\
\hline Спосіб обрахунку & \multicolumn{4}{|c|}{ Повнопрофільний } \\
\hline $2 \theta \mathrm{i} \sin \theta / \lambda_{\text {(1ancs) }}$ & \multicolumn{4}{|c|}{$100.0 \quad 0.497$} \\
\hline Кількість атомних позицій & 4 & 4 & 4 & 4 \\
\hline $\mathrm{R}_{\mathrm{I}}$ & 0.1058 & 0.0815 & 0.0896 & 0.0619 \\
\hline $\mathrm{R}_{\mathrm{p}}$ & 0.2653 & 0.2641 & 0.2074 & 0.1586 \\
\hline Кількість вільних параметрів & 14 & 14 & 14 & 14 \\
\hline
\end{tabular}



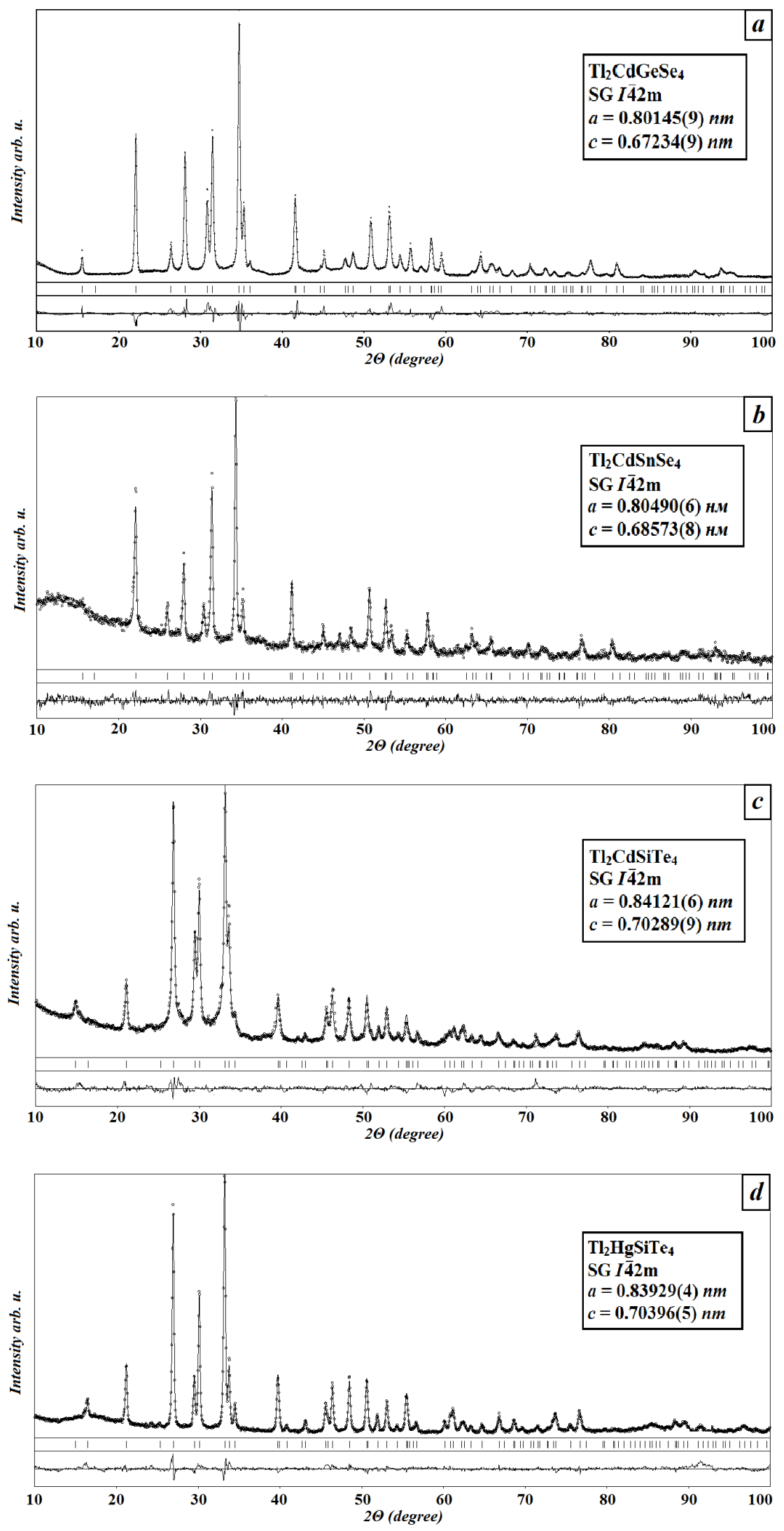

Рис. 3. Експериментальні (кола) та теоретичні (лінії) дифракційні профілі та їх різницева для сполук: $a-\mathrm{Tl}_{2} \mathrm{CdGeSe}_{4}$, $b-\mathrm{Tl}_{2} \mathrm{CdSnSe}_{4}, c-\mathrm{Tl}_{2} \mathrm{CdSiTe}{ }_{4}, d-\mathrm{Tl}_{2} \mathrm{HgSiTe}_{4}$ 
Залежність об'єму просторової гратки та розрахованої густини від молярних мас в ізоструктурних сполуках $\mathrm{Tl}_{2} \mathrm{~B}^{\mathrm{II}} \mathrm{D}^{\mathrm{IV}} \mathrm{X}_{4}$

\begin{tabular}{|c|c|c|c|c|c|}
\hline Сполука & ПГГ & М, г/моль & V, нм $^{3}$ & Розрахункова густина, г/см ${ }^{3}$ & Л-pa \\
\hline $\mathrm{Tl}_{2} \mathrm{HgSnS}_{4}$ & \multirow{14}{*}{$I-42 m$} & 856.1 & 0.4138 & 6.871 & {$[20]$} \\
\hline $\mathrm{Tl}_{2} \mathrm{HgSnS}_{4}$ & & 909.8 & 0.4319 & 6.995 & {$[*$} \\
\hline $\mathrm{Tl}_{2} \mathrm{HgSiSe}_{4}$ & & 953.5 & 0.4284 & 7.390 & [21] \\
\hline $\mathrm{Tl}_{2} \mathrm{CdSnSe}_{4}$ & & 955.9 & 0.4439 & 7.144 & {$[24]$} \\
\hline $\mathrm{Tl}_{2} \mathrm{HgGeSe}_{4}$ & & 998.0 & 0.4322 & 7.716 & {$[22]$} \\
\hline $\mathrm{Tl}_{2} \mathrm{MnGeTe}_{4}$ & & 1046.7 & 0.4912 & 7.080 & [20] \\
\hline $\mathrm{Tl}_{2} \mathrm{HgSnSe}_{4}$ & & 1044.1 & 0.4452 & 7.787 & [21] \\
\hline $\mathrm{Tl}_{2} \mathrm{CdSiTe}_{4}$ & & 1059.6 & 0.4974 & 7.075 & {$[*$} \\
\hline $\mathrm{Tl}_{2} \mathrm{MnSnTe}_{4}$ & & 1092.8 & 0.5076 & 7.150 & [20] \\
\hline $\mathrm{Tl}_{2} \mathrm{CdGeTe}_{4}$ & & 1104.2 & 0.4973 & 7.370 & [20] \\
\hline $\mathrm{Tl}_{2} \mathrm{HgSiTe}_{4}$ & & 1147.8 & 0.4959 & 7.687 & {$[*$} \\
\hline $\mathrm{Tl}_{2} \mathrm{CdSnTe}_{4}$ & & 1150.3 & 0.5123 & 7.460 & [20] \\
\hline $\mathrm{Tl}_{2} \mathrm{HgGeTe}_{4}$ & & 1192.4 & 0.4937 & 8.020 & {$[20]$} \\
\hline $\mathrm{Tl}_{2} \mathrm{HgSnTe}_{4}$ & & 1238.5 & 0.5047 & 8.150 & {$[20]$} \\
\hline
\end{tabular}

*- дана робота

Таблиця 4 талієвмісних тетрарних сполуках, взятих для

Результати розшифрування кристалічної структури сполуки $\mathrm{Tl}_{2} \mathrm{CdGe}_{3} \mathrm{Se}_{8}$

\begin{tabular}{|c|c|}
\hline Сполука & $\mathrm{Tl}_{2} \mathrm{CdGe}_{3} \mathrm{Se}_{8}$ \\
\hline Просторова група & $P 2_{1} 2_{1} 2_{1}$ \\
\hline \multicolumn{2}{|l|}{ Параметри комірки: } \\
\hline$a(\mathrm{HM})$ & $0,76023(9)$ \\
\hline$b$ (нм) & $1,2071(2)$ \\
\hline$c$ (нм) & $1,7474(2)$ \\
\hline$V\left(\mathrm{HM}^{3}\right)$ & $1,6036(6)$ \\
\hline$F(000)$ & 2312.0 \\
\hline Кількість атомних позицій & 56.0 \\
\hline 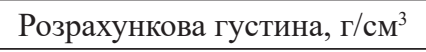 & $5.676(2)$ \\
\hline Коефіцієнт абсорбції, 1/см & 794.03 \\
\hline $\begin{array}{l}\text { Випромінювання; } \\
\text { довжина хвилі, нм }\end{array}$ & CuK 1.54185 \\
\hline Дифрактометр & Порошковий \\
\hline Спосіб обрахунку & Повнопрофільний \\
\hline Кількість атомних позицій & 14 \\
\hline Кількість вільних параметрів & 58 \\
\hline $2 \theta \mathrm{i} \sin \theta / \lambda_{\text {(макс) }}$ & 100.050 .497 \\
\hline h(мін), k(мін), l(мін) & 000 \\
\hline h(макс), k(макс), l(макс) & 71117 \\
\hline$R_{I} \mathrm{i} R_{\mathrm{w}}$ & 0.12040 .2783 \\
\hline Скалярний фактор & $1.98(6)$ \\
\hline Вісь і параметр текстури & {$\left[\begin{array}{lll}0 & 1 & 0\end{array}\right] 0.134(5)$} \\
\hline
\end{tabular}

Зменшення, очевидно, можна пояснити ефектом $\mathrm{f}$-стиснення в атомі $\mathrm{Hg}$. При заміні $\mathrm{Ge} \rightarrow \mathrm{Sn}$ у нових, та аналогічних до них за структурою порівняння $\left(\mathrm{Tl}_{2} \mathrm{Cd}(\mathrm{Hg}) \mathrm{GeSe}_{4}\left(\mathrm{Te}_{4}\right) \rightarrow \mathrm{Tl}_{2} \mathrm{Cd}(\mathrm{Hg})\right.$ $\mathrm{SnSe}_{4}\left(\mathrm{Te}_{4}\right)$ та $\left.\mathrm{Tl}_{2} \mathrm{MnGeTe}_{4} \rightarrow \mathrm{Tl}_{2} \mathrm{MnSnTe}_{4}\right)$ спостерігається закономірне зростання об'єму просторової гратки. Однак заміна $\mathrm{Si} \rightarrow \mathrm{Ge}$ у сполуках $\mathrm{Tl}_{2} \mathrm{Cd}(\mathrm{Hg}) \mathrm{Si}\left(\mathrm{Te}_{4}\right) \rightarrow \mathrm{Tl}_{2} \mathrm{Cd}(\mathrm{Hg}) \mathrm{Ge}\left(\mathrm{Te}_{4}\right)$ призводить до зменшення комірки через ефект d-стиснення в атомі Ge. У всіх випадках при зміні $\mathrm{S} \rightarrow \mathrm{Se} \rightarrow$ Tе суттєво збільшуються розміри комірки. Розрахована густина суттєво зростає 3 молярною масою у всіх випадках заміщення дво-, чотири-, або шестивалентного елемента.

Тетрарна сполука $\mathrm{Tl}_{2} \mathrm{CdGe}_{3} \mathrm{Se}_{8}$, що утворюється на перерізі $\mathrm{Tl}_{2} \mathrm{CdGeSe}_{4}-\mathrm{GeSe}_{2}$ квазіпотрійної системи $\mathrm{Tl}_{2} \mathrm{Se}-\mathrm{CdSe}-\mathrm{GeSe}_{2}$, кристалізується в нецентросиметричній ПГ $P 22_{1} 2_{1}$ (CT $\mathrm{Cs}_{2} \mathrm{HgGe}_{3} \mathrm{Se}_{8}$ ). На рис. 4 наведені іiі експериментальні, теоретичні та різницеві між ними рентгенодифракційні спектри.

Координати та кристалографічні параметри у структурі $\mathrm{Tl}_{2} \mathrm{CdGe}_{3} \mathrm{Se}_{8}$ представлені в табл. 4.

Отже, за результатами рентгенофазовим аналізом побудовано ізотермічні перерізи систем $\mathrm{Tl}_{2} \mathrm{Se}-\mathrm{CdSe}-\mathrm{Ge}(\mathrm{Sn}) \mathrm{Se}_{2}$ при 570 К. Підтверджено існування сполуки $\mathrm{Tl}_{2} \mathrm{CdSnSe}_{4}$. Встановлено утворення та розшифровано кристалічну структуру чотирьох тетрарних сполук: $\mathrm{Tl}_{2} \mathrm{CdGe}_{3} \mathrm{Se}_{8}$ та трьох ізоструктурних халькогенідів: $\mathrm{Tl}_{2} \mathrm{CdGeSe}_{4}, \mathrm{Tl}_{2} \mathrm{CdSiTe}_{4}, \mathrm{Tl}_{2} \mathrm{HgSiTe}_{4}$. $\mathrm{Tl}_{2} \mathrm{CdGe}_{3} \mathrm{Se}_{8}$ кристалізується в тригональній ПГ $P 22_{1} 2_{1}$ з параметрами: $a=0,7.6023(9)$, 


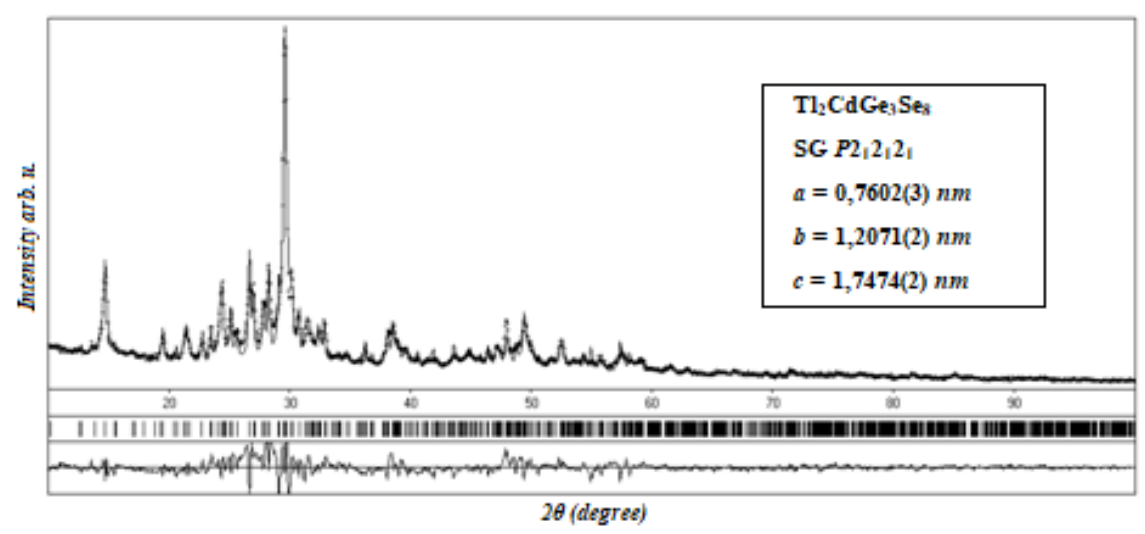

\section{Рис 4. Експериментальні (кола) та теоретичні (лініi) дифракційні профілі та їх різницева для сполуки $\mathrm{Tl}_{2} \mathrm{CdGe}_{3} \mathrm{Se}_{8}$}

$b=1,2071(2), c=1,7474(2)$ нм. $\mathrm{Tl}_{2} \mathrm{~B}^{\mathrm{II}} \mathrm{D}^{\mathrm{IV}} \mathrm{X}_{4}$ кристалізуються в тетрагональній структурі з ПГ I-42m. Параметри комірок сполук: $a=0.80145(9)$, $c=0.67234(9) \mathrm{nm}\left(\mathrm{Tl}_{2} \mathrm{CdGeSe}_{4}\right) ; a=0.8049(6)$, $c=0.68573(8) \mathrm{nm}\left(\mathrm{Tl}_{2} \mathrm{CdSnSe}_{4}\right) ; a=0.84121(6)$, $c=0.70289(9) \mathrm{nm}\left(\mathrm{Tl}_{2} \mathrm{CdSiTe}_{4}\right) ; a=0.83929(4)$, $c=0.70396(5) \mathrm{nm}\left(\mathrm{Tl}_{2} \mathrm{HgSiTe}_{4}\right)$. Розглянуто залежність об'єму просторової гратки та розрахованої густини від молярної маси у одинадцяти відомих раніше та трьох нововиявлених ізоструктурних (ПГ $I-42 m$ ) сполуках $\mathrm{Tl}_{2} \mathrm{~B}^{\mathrm{II}} \mathrm{D}^{\mathrm{IV}} \mathrm{X}_{4}$. Отримані сполуки, кристалізуючись в нецентросиметричній структурі представляють інтерес для подальших досліджень.

\section{ЛIТЕРАТУРА:}

1. Piskach L.V., Parasyuk O.V., Olekseyuk I.D. The phase equilibria in the quasi-ternary $\mathrm{Cu}_{2} \mathrm{~S}-\mathrm{CdS}-\mathrm{SnS}_{2}$ system. J. Alloys Compds. 1998. 279(2). P. 142-152.

2. Kanno R., Hata T., Kawamoto Y., Irie M. Synthesis of a new lithium ionic conductor, thio-LISICON-lithium germanium sulfide system. Solid State Ionics, 2000. 130(1-2). P. 97-104.

3. Parasyuk O.V., Gulay L.D., Piskach L.V., Olekseyuk I.D. The $\mathrm{Ag}_{2} \mathrm{Se}-\mathrm{CdSe}-\mathrm{SnSe}_{2}$ system at $670 \mathrm{~K}$ and the crystal structure of the $\mathrm{Ag}_{2} \mathrm{CdSnSe}_{4}$ compound. J. Alloys Compds. 2002. 335(1-2). P. 176-180.

4. Parasyuk O.V., Gulay L.D., Piskach L.V., Kumanska Yu.O. The $\mathrm{Ag}_{2} \mathrm{Se}-\mathrm{HgSe}-\mathrm{SnSe}_{2}$ system and the crystal structure of the $\mathrm{Ag}_{2} \mathrm{HgSnSe}_{4}$ compound. J. Alloys Compds. 2002. 339(1-2). P. 140-143.

5. Parasyuk O.V., Gulay L.D., Romanyuk Y.E., Olekseyuk I.D, Piskach L.V. The $\mathrm{Ag}_{2} \mathrm{Se}-\mathrm{HgSe}-\mathrm{GeSe}_{2}$ system and crystal structures of the compounds. J. Alloys Compds. 2003. 351(1-2). P. 135-144.

6. Parasyuk O.V., Chykhrij S.I., Bozhko V.V. Piskach L.V., Bogdanyuk M.S., Olekseyuk I.D., Bulatetska L.V., Pekhnyo V.I. Phase diagram of the $\mathrm{Ag}_{2} \mathrm{~S}-\mathrm{HgS}-\mathrm{SnS}_{2}$ system and single crystal preparation, crystal structure and properties of $\mathrm{Ag}_{2} \mathrm{HgSnS}_{4}$. J. Alloys Compds. 2005. 399(1-2). P. 32-37.

7. Olekseyuk I.D., Piskach L.V., Zhbankov O.Y., Parasyuk O.V., Kogut Yu.M. Phase diagrams of the quasi-binary systems $\mathrm{Cu}_{2} \mathrm{~S}_{-} \mathrm{SiS}_{2}$ and $\mathrm{Cu}_{2} \mathrm{SiS}_{3}-\mathrm{PbS}$ and the crystal structure of the new quaternary compound $\mathrm{Cu}_{2} \mathrm{PbSiS}_{4}$. J. Alloys Compds. 2005. 399(1-2). P. 149-154.

8. Parasyuk O.V., Fedorchuk A.O., Kogut Y.M. et al., The $\mathrm{Ag}_{2} \mathrm{~S}-\mathrm{ZnS}-\mathrm{GeS}_{2}$ system: Phase diagram, glass-formation region and crystal structure of $\mathrm{Ag}_{2} \mathrm{ZnGeS}_{4}$. J. Alloys Compds. 2010. 500(1). P. $26-29$.

9. Kogut Y., Fedorchuk A., Zhbankov O., Romanyuk Ya., Kityk I., Piskach L., Parasyuk O. Isothermal section of the $\mathrm{Ag}_{2} \mathrm{~S}-\mathrm{PbS}-\mathrm{GeS}_{2}$ system at $300 \mathrm{~K}$ and the crystal structure of $\mathrm{Ag}_{2} \mathrm{PbGeS}_{4}$. J. Alloys Compds. 2011. 509(11). P. 4264-4267.

10. Schumer B. N., Downs R. T., Domanik Kenneth J., Andrade M., Origlieri M. J. Pirquitasite, $\mathrm{Ag}_{2} \mathrm{ZnSnS}_{4}$. Acta Cryst. 2013. 69(2). P. i8-i9.

11. Zhang J.-H., Clark D. J., Weiland A., Stoyko S. S., Soo Kim Y., Jang J. I., Aitken J. A. Li ${ }_{2} \mathrm{CdGeSe}_{4}$ and $\mathrm{Li}_{2} \mathrm{CdSnSe}_{4}$ : biaxial nonlinear optical materials with strong infrared second-order responses and laser-induced damage thresholds influenced by photoluminescence. Inorg. Chem. Front. 2017. 4. P. 1472-1484.

12. He J., Guo Y., Huang W., Zhang X., Yao J., Zhai T., Huang F. Synthesis, Crystal Structure, and Optical Properties of Noncentrosymmetric $\mathrm{Na}_{2} \mathrm{ZnSnS}_{4}$. Inorg. Chem. 2018, 57(16), P. 9918-9924.

13. Brik M.G., Parasyuk O.V., Myronchuk G.L., Kityk I.V. Specific features of band structure and optical anisotropy of $\mathrm{Cu}_{2} \mathrm{CdGeSe}_{4}$ quaternary compounds. Mat. Chem. Phys. 2014. 147. P. 155-161. 
14. Rincón C., Quintero M.E., Moreno P.Ch., Quintero E., Henao J.A., Macías M.A. Raman spectrum of $\mathrm{Cu}_{2} \mathrm{CdSnSe}_{4}$ stannite structure semiconductor compound. Superlattices and Microstruct. 2015. 88. P. 99-103.

15. Kogut Y., Khyzhun O.Y., Parasyuk O.V., Reshak A.H., Lakshminarayana G., Kityk I.V, Piasecki M. Electronic spectral parameters and IR nonlinear optical features of novel $\mathrm{Ag}_{0.5} \mathrm{~Pb}_{1.75} \mathrm{GeS}_{4}$ crystal. J. Crystal Growth. 2012. 354(1). P. 142-146.

16. Reshak A.H., Kogut Y.M., Fedorchuk A.O., Zamuruyeva O.V., Myronchuk G.L., Parasyuk O.V., Kamarudin H.,

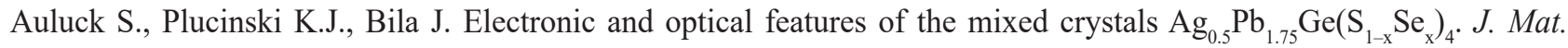
Chem. C. 2013. 1(31). P. 4667-4675.

17. Litvinchuk A.P., Dzhagan V.M., Yukhymchuk V.O., Valakh M.Ya., Babichuk I.S., Parasyuk O.V., Piskach L.V., Gordan O.D., Zahn D.R. T. Electronic structure, optical properties, and lattice dynamics of orthorhombic $\mathrm{Cu}_{2} \mathrm{CdGeS}_{4}$ and $\mathrm{Cu}_{2} \mathrm{CdSiS}_{4}$ semiconductors. Phys. Rev B. 2014. 90(16). P. 165-201.

18. Zhang Y., Sun X., Zhang P., Yuan X., Huang F., Zhang W. Structural properties and quasiparticle band structures of Cu-based quaternary semiconductors for photovoltaic applications. J. Appl. Phys. 2012. 111(6). P. 63709.

19. Huang Y., Wu K., Cheng J., Zhihua Y., Pan Sh. $\mathrm{Li}_{2} \mathrm{ZnGeS}_{4}$ : a promising diamond-like infrared nonlinear optical material with high laser damage threshold and outstanding second-harmonic generation response. Dalton Trans. 2019. 48(14). P. 4484-4488.

20. Eulenberger G. Darstellung und Kristallstruktur des Dithallium(I) blei(II)- tetrathiogermanats(IV) $\mathrm{Tl}_{2} \mathrm{PbGeS}_{4} /$ Preparation and Crystal Structure of Dithallium(I) Lead(II) Tetrathiogermanate(IV). Z. Naturforsch. 1980. 35 . P. $335-339$.

21. McGuire M.A., Scheidemantel Th.J., Badding J.V., Badding John V., DiSalvo F. J. Tl $\mathrm{AXTe}_{4}(\mathrm{~A}=\mathrm{Cd}, \mathrm{Hg}, \mathrm{Mn}$; $\mathrm{X}=\mathrm{Ge}, \mathrm{Sn})$ : Crystal Structure, Electronic Structure, and Thermoelectric Properties. Chem. Mater. 2005. 17. P. 6186-6191.

22. Mozolyuk M.Yu., Piskach L.V., Fedorchuk A.O., Olekseyuk I.D., Parasyuk O.V. Physico-chemical interaction in the $\mathrm{Tl}_{2} \mathrm{Se}-\mathrm{HgSe}-\mathrm{D}^{\mathrm{IV}} \mathrm{Se}_{2}$ systems (D $\left.{ }^{\mathrm{IV}}-\mathrm{Si}, \mathrm{Sn}\right)$. Mater. Res. Bull. 2012. 47. P. 3830-3834.

23. Mozolyuk M.Yu., Piskach L.V., Fedorchuk A.O., Olekseyuk I.D., Parasyuk O.V. The Tl 2 Se-HgSe-GeSe ${ }_{2}$ system and the crystal structure of $\mathrm{Tl}_{2} \mathrm{HgGeSe}_{4}$. Chem. Met. Alloys. 2013. 6. P. 55-62.

24. Piskach L.V., Mozolyuk M.Yu., Fedorchuk A.O., Olekseyuk I.D., Parasyuk O.V. Phase equilibria in the $\mathrm{Tl}_{2} \mathrm{~S}-\mathrm{HgS}-\mathrm{SnS}_{2}$ system at $520 \mathrm{~K}$ and crystal structure of $\mathrm{Tl}_{2} \mathrm{HgSnS}_{4}$. Chem. Met. Alloys. 2017. 10. P. 136-141.

25. Selezen A.O., Piskach L.V., Parasyuk O.V., Olekseyuk I.D. The $\mathrm{Tl}_{2} \mathrm{SnSe}_{3}$-CdSe System and the Crystal Structure of the $\mathrm{Tl}_{2} \mathrm{CdSnSe}_{4}$ compound. J. Phase Equilib. Diffus. 2019. 40, 6. P. 797-801.

26. Mozolyuk M.Yu., Piskach L.V., Fedorchuk A.O., Parasyuk O.V., Khyzhun O.Y. The $\mathrm{Tl}_{2} \mathrm{~S}-\mathrm{PbS}-\mathrm{SiS}{ }_{2}$ system and the crystal and electronic structure of quaternary chalcogenide $\mathrm{Tl}_{2} \mathrm{PbSiS}_{4}$. 2017. Mat. Chem. Phys. 195. P. $132-142$.

27. Цісар О., Піскач Л., Бабіжецький В., Левицький В., Котур Б., Марушко Л., Олексеюк І., Парасюк О. Фазові рівноваги в системі $\mathrm{Tl}_{2} \mathrm{Se}-\mathrm{In}_{2} \mathrm{Se}_{3}-\mathrm{GeSe}_{2}$ при 520 К. Вісн. Львів. у-ту. Сер. хімічна. 2018. 59(10). С. 46-52.

28. Davydyuk G.E., Piasecki M., Parasyuk O.V., Myronchuk G.L., Fedorchuk A. O., Danylchuk S.P., Piskach L.V., Mozolyuk M.Yu., AlZayed N. 2013. Opt. Mater. 35(12). P. 2514-2518.

29. Khyzhun O.Y., Fedorchuk A.O., Kityk I.V., Piasecki M., Mozolyuk M.Yu., Piskach L.V., Parasyuk O.V., ElNaggar A.M., Albassam A.A., Karasinski P. Electronic structure and laser induced piezoelectricity of a new quaternary compound TlinGe $\mathrm{S}_{8}$. Mat. Chem. Phys. 2018. 204. P. 336-344.

30. Myronchuk G.L., Zamurueva O.V., Parasyuk O.V., Piskach L.V., Fedorchuk A.O., AlZayed N.S., El-Naggar A.M., Ebothe J., Lis M., Kityk I.V. Structural and optical properties of novel optoelectronic $\mathrm{Tl}_{1-\mathrm{x}} \mathrm{In}_{1-\mathrm{x}} \mathrm{Si}_{\mathrm{x}} \mathrm{Se}_{2}$ single crystals. J. Mat. Sci.: Mat. in Electr. 25(7). P. 3226-3232.

31. Myronchuk G.L., Davydyuk G.E., Parasyuk O.V., Khyzhun O.Y., Andrievski R.A., Fedorchuk A.O., Danylchuk S.P., Piskach L.V., Mozolyuk M.Y. Tl ${ }_{1-x} \operatorname{In}_{1-x} \mathrm{Sn}_{\mathrm{x}} \mathrm{Se}_{2}(x=0,0.1,0.2,0.25)$ single-crystalline alloys as promising non-linear optical materials. J. Mat. Sci.: Mat. in Electr. 2013. 24(9). P. 3555-3563.

32. Абрикосов Н.Х., Банкина В.Ф., Порецкая Л.В. [и др.]. Полупроводниковые халькогениды и сплавы на их основе. Москва : Наука, 1975. С. 219.

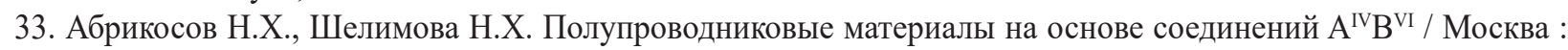
Наука, 1975. C. 195.

34. Glukh A.S., Sabov M.Yu., Barchii I.E., Tsigika V.V., Sidei V.I. Formation of ternary compounds in the $\mathrm{Tl}_{2} \mathrm{Se}-\mathrm{GeSe}{ }_{2}$ system. Inorgan. Mater. 2009. 45. P. 1172-1176.

35. Houenou P., Eholie R., Etude du systeme $\mathrm{SnSe}_{2}-\mathrm{Tl}_{2} \mathrm{Se}$. Acad. Sci. Paris. 1976. 283. 16. P. 731-733.

36. Лазарев В.Б., Переш Е.Ю., Староста В.И., Мудрый В.В. Фазовые равновесия и свойства соединений в системах $\mathrm{Tl}_{2} \mathrm{~S}(\mathrm{Se})-\mathrm{SnS}_{2}\left(\mathrm{Se}_{2}\right)$. Журн. неорг. химии. 1985. 30(6). С. 1502-1506.

37. Mucha I., Wiglusz K., Sztuba Z., Gaweł W. Solid-liquid equilibria in the quasi-binary thallium(I) selenide-tin(IV) selenide system. Comp. Coupl. Phase Diagr. and Т И.H. hermochem. 2009. 33. P. 545-549.

38. Один В.В., Гринко В.В., Новоселова А.В. Р-T-X фазовая диаграмма системы CdSe-GeSe. Журнал неорганической химии. 1986. 31(5). С. 1274-1277. 
39. Стасова М.М., Вайнштейн Б.К. Электронографическое определение структуры $\mathrm{Tl}_{2} \mathrm{Se}$. Кристаллография. 1958. 3(2). C. 141-147.

40. Dittmar G., Schafer H. Die Kristallstruktur von germanium diselenid. Acta Cryst. B. 1976. 32. P. 2726-2728.

41. Busch G., Frohlich C., Hulliger F., Steimeier E. Structur, elektrische und thermoelektrische Eigenschaften von $\mathrm{SnSe}_{2}$. Helv. Phys. Acta. 1961. 34. P. 359-368.

42. Glukh O.S., Sabov M.Yu., Barchij I.E., Pavlyuk V.V., Marciniak B. Crystal structure of the $\mathrm{Tl}_{4} \mathrm{GeSe}_{4}$ ternary compound. Chem. Met. Alloys. 2009 (2). P. 10-14.

43. Eulenberger G. Ternäre Thalliumchalkogenide mit $\mathrm{Tl}_{4} \mathrm{Ge}_{2} \mathrm{~S}_{6}$-Struktur. Monatsh. Chem. 1982. 113. P. 859-867.

44. Eulenberger $\mathrm{G}_{4} \mathrm{Tl}_{4} \mathrm{Ge}_{4} \mathrm{Se}_{10}$, ein Thallium(1)selenogermanat mit adamantananalogem Anion $\left[\mathrm{Ge}_{4} \mathrm{Se}_{10}\right]^{4-/} \mathrm{Tl}_{4} \mathrm{Ge}_{4} \mathrm{Se}_{10}$, a Thallium(I) Selenogermanate with the Adamantane-Like Anion $\left[\mathrm{Ge}_{4} \mathrm{Se}_{10}\right]^{4-}$. Z. Naturforsch. 1981. 36. P. 521-523.

45. Akinocho G., Houenou P., Oyetola S., Eholie R., Jumas J. C., Olivier-Fourcade J., Maurin M. Étude structurale de $\mathrm{Tl}_{4} \mathrm{SnSe}_{4}$. J. Solid State Chem. 1991. 93(2). P. 336-340.

46. Jaulmes S., Houenou P., Structure cristalline du seleniure d'etain(IV) et de thallium(I): $\mathrm{Tl}_{2} \mathrm{SnSe}_{3}$. Mater. Res. Bull. 1980. 15(7). P. 911-915.

47. Henao J.A., Delgado J.M., Quintero M., X-ray powder diffraction data and structural study of $\mathrm{Fe}_{2} \mathrm{GeSe}_{4}$. Powder Diffr. 1998. 13(4) P. 202-209.

48. Akselrud L.G., Zavalii P.Yu., Grin Yu. et al., J. WinCSD: software package for crystallographic calculations (Version 4). J. Appl. Cryst. 2014. 47. P. 803-805.

49. Ketelaar J.A., t'Hart W.H., Moerel M., Polder D. The Crystal Structure of TISe, Thallous Thallic or Thallosic Selenide. Z. Kristallog. A. 1939. 101 P. 396-404.

50. Müller D., Eulenberger G., Hahn H. Über ternäre Thalliumchalkogenide mit Thalliumselenidstruktur. Z. Anorg. Allg. Chem, 1973. 398. P. 207-220.

\section{REFERENCES:}

1. Piskach, L.V., Parasyuk, O.V., Olekseyuk, I.D. (1998). The phase equilibria in the quasi-ternary $\mathrm{Cu}_{2} \mathrm{~S}-\mathrm{CdS}-$ $\mathrm{SnS}_{2}$ system. J. Alloys Compds, 279(2). 142-152.

2. Kanno, R., Hata, T., Kawamoto, Y., Irie, M. (2000). Synthesis of a new lithium ionic conductor, thio-LISICONlithium germanium sulfide system. Solid State Ionics, 130(1-2), 97-104.

3. Parasyuk, O.V., Gulay, L.D., Piskach, L.V., Olekseyuk, I.D. (2002). The $\mathrm{Ag}_{2} \mathrm{Se}-\mathrm{CdSe}-\mathrm{SnSe}_{2}$ system at $670 \mathrm{~K}$ and the crystal structure of the $\mathrm{Ag}_{2} \mathrm{CdSnSe}_{4}$ compound. J. Alloys Compds. 335(1-2), 176-180.

4. Parasyuk, O.V., Gulay, L.D., Piskach, L.V., Kumanska, Yu.O. (2002). The $\mathrm{Ag}_{2} \mathrm{Se}-\mathrm{HgSe}-\mathrm{SnSe}_{2}$ system and the crystal structure of the $\mathrm{Ag}_{2} \mathrm{HgSnSe}_{4}$ compound. J. Alloys Compds. 339(1-2), 140-143.

5. Parasyuk, O.V., Gulay, L.D., Romanyuk, Y.E., Olekseyuk, I.D, Piskach, L.V. (2003). The $\mathrm{Ag}_{2} \mathrm{Se}-\mathrm{HgSe}-\mathrm{GeSe}_{2}$ system and crystal structures of the compounds. J. Alloys Compds. 351(1-2), 135-144.

6. Parasyuk, O.V., Chykhrij, S.I., Bozhko, V.V. Piskach, L.V., Bogdanyuk, M.S., Olekseyuk, I.D., Bulatetska, L.V., Pekhnyo, V.I. (2005). Phase diagram of the $\mathrm{Ag}_{2} \mathrm{~S}-\mathrm{HgS}-\mathrm{SnS}_{2}$ system and single crystal preparation, crystal structure and properties of $\mathrm{Ag}_{2} \mathrm{HgSnS}_{4}$. J. Alloys Compds. 399(1-2), 32-37.

7. Olekseyuk, I.D., Piskach, L.V., Zhbankov, O.Y., Parasyuk, O.V., Kogut, Yu.M. (2005). Phase diagrams of the quasi-binary systems $\mathrm{Cu}_{2} \mathrm{~S}-\mathrm{SiS}_{2}$ and $\mathrm{Cu}_{2} \mathrm{SiS}_{3}-\mathrm{PbS}$ and the crystal structure of the new quaternary compound $\mathrm{Cu}_{2} \mathrm{PbSiS}_{4}$. J. Alloys Compds. 399(1-2), 149-154.

8. Parasyuk, O.V., Fedorchuk, A.O., Kogut, Y.M. et al. (2010) The $\mathrm{Ag}_{2} \mathrm{~S}-\mathrm{ZnS}-\mathrm{GeS}_{2}$ system: Phase diagram, glassformation region and crystal structure of $\mathrm{Ag}_{2} \mathrm{ZnGeS}_{4}$. J. Alloys Compds. 500(1), 26-29.

9. Kogut, Y., Fedorchuk, A., Zhbankov, O., Romanyuk, Ya., Kityk, I., Piskach, L., Parasyuk, O. (2011). Isothermal section of the $\mathrm{Ag}_{2} \mathrm{~S}-\mathrm{PbS}-\mathrm{GeS}_{2}$ system at $300 \mathrm{~K}$ and the crystal structure of $\mathrm{Ag}_{2} \mathrm{PbGeS}_{4}$. J. Alloys Compds. 509(11), 4264-4267.

10. Schumer, B.N., Downs, R.T., Domanik, Kenneth J., Andrade, M., Origlieri M.J. (2013). Pirquitasite, $\mathrm{Ag}_{2} \mathrm{ZnSnS}_{4}$. Acta Cryst. 69(2), i8-i9.

11. Zhang, J.-H., Clark, D.J., Weiland, A., Stoyko, S.S., Soo Kim Y., Jang, J.I., Aitken, J.A. (2017). $\mathrm{Li}_{2} \mathrm{CdGeSe}_{4}$ and $\mathrm{Li}_{2} \mathrm{CdSnSe}_{4}$ : biaxial nonlinear optical materials with strong infrared second-order responses and laser-induced damage thresholds influenced by photoluminescence. Inorg. Chem. Front., 4, 1472-1484.

12. He, J., Guo, Y., Huang, W., Zhang, X., Yao, J., Zhai, T., Huang, F. (2018). Synthesis, Crystal Structure, and Optical Properties of Noncentrosymmetric $\mathrm{Na}_{2} \mathrm{ZnSnS}_{4}$. Inorg. Chem. 57(16), 9918-9924.

13. Brik, M.G., Parasyuk, O.V., Myronchuk, G.L., Kityk, I.V. (2014). Specific features of band structure and optical anisotropy of $\mathrm{Cu}_{2} \mathrm{CdGeSe}_{4}$ quaternary compounds. Mat. Chem. Phys, 147, 155-161.

14. Rincón, C., Quintero, M.E., Moreno, P.Ch., Quintero, E., Henao, J.A., Macías, M.A. (2015). Raman spectrum of $\mathrm{Cu}_{2} \mathrm{CdSnSe}_{4}$ stannite structure semiconductor compound. Superlattices and Microstruct. 88, 99-103. 
15. Kogut, Y., Khyzhun, O.Y., Parasyuk, O.V., Reshak,A.H., Lakshminarayana, G., Kityk, I.V, Piasecki M. (2012). Electronic spectral parameters and IR nonlinear optical features of novel $\mathrm{Ag}_{0.5} \mathrm{~Pb}_{175} \mathrm{GeS}_{4}$ crystal. J. Crystal Growth, 354(1), 142-146.

16. Reshak, A.H., Kogut, Y.M., Fedorchuk, A.O., Zamuruyeva, O.V., Myronchuk, G.L., Parasyuk, O.V., Kamarudin,

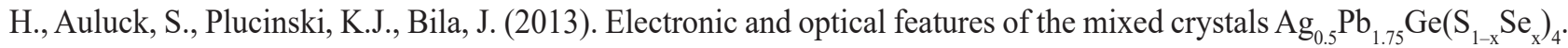
J. Mat. Chem. C, 1(31), 4667-4675.

17. Litvinchuk, A.P., Dzhagan, V.M., Yukhymchuk, V.O., Valakh, M.Ya., Babichuk, I.S., Parasyuk, O.V., Piskach, L.V., Gordan, O.D., Zahn, D.R.T. (2014). Electronic structure, optical properties, and lattice dynamics of orthorhombic $\mathrm{Cu}_{2} \mathrm{CdGeS}_{4}$ and $\mathrm{Cu}_{2} \mathrm{CdSiS}_{4}$ semiconductors. Phys. Rev B. 90(16).

18. Zhang, Y., Sun, X., Zhang, P., Yuan, X., Huang, F., Zhang, W. (2012). Structural properties and quasiparticle band structures of Cu-based quaternary semiconductors for photovoltaic applications. J. Appl. Phys, 111(6).

19. Huang, Y., Wu, K., Cheng, J., Zhihua, Y., Pan, Sh. (2019). Li $\mathrm{ZnGeS}_{4}$ : a promising diamond-like infrared nonlinear optical material with high laser damage threshold and outstanding second-harmonic generation response. Dalton Trans, 48(14), 4484-4488.

20. Eulenberger, G. (1980). Darstellung und Kristallstruktur des Dithallium(I) blei(II)- tetrathiogermanats(IV) $\mathrm{Tl}_{2} \mathrm{PbGeS}_{4} /$ Preparation and Crystal Structure of Dithallium(I) Lead(II) Tetrathiogermanate(IV). Z. Naturforsch, 35, 335-339.

21. McGuire, M.A., Scheidemantel, Th.J., Badding, J.V., Badding, John V., DiSalvo, F.J. (2005). $\mathrm{Tl}_{2} \mathrm{AXTe}_{4}(\mathrm{~A}=\mathrm{Cd}$, $\mathrm{Hg}, \mathrm{Mn} ; \mathrm{X}=\mathrm{Ge}, \mathrm{Sn})$ : Crystal Structure, Electronic Structure, and Thermoelectric Properties. Chem. Mater, 17, 6186-6191.

22. Mozolyuk, M.Yu., Piskach, L.V., Fedorchuk, A.O., Olekseyuk, I.D., Parasyuk, O.V. (2012). Physico-chemical interaction in the $\mathrm{Tl}_{2} \mathrm{Se}-\mathrm{HgSe}-\mathrm{D}^{\mathrm{IV}} \mathrm{Se}_{2}$ systems ( $\left.\mathrm{D}^{\mathrm{IV}}-\mathrm{Si}, \mathrm{Sn}\right)$. Mater. Res. Bull, 47, 3830-3834.

23. Mozolyuk, M.Yu., Piskach, L.V., Fedorchuk, A.O., Olekseyuk, I.D., Parasyuk, O.V. (2013). The $\mathrm{Tl}_{2} \mathrm{Se}-\mathrm{HgSe}-$ $\mathrm{GeSe}_{2}$ system and the crystal structure of $\mathrm{Tl}_{2} \mathrm{HgGeSe}_{4}$. Chem. Met. Alloys, 6, 55-62.

24. Piskach, L.V., Mozolyuk, M.Yu., Fedorchuk, A.O., Olekseyuk, I.D., Parasyuk O.V. (2017). Phase equilibria in the $\mathrm{Tl}_{2} \mathrm{~S}-\mathrm{HgS}-\mathrm{SnS}_{2}$ system at $520 \mathrm{~K}$ and crystal structure of $\mathrm{Tl}_{2} \mathrm{HgSnS}_{4}$. Chem. Met. Alloys, 10, 136-141.

25. Selezen, A.O., Piskach, L.V., Parasyuk, O.V., Olekseyuk, I.D. (2019). The $\mathrm{Tl}_{2} \mathrm{SnSe}_{3}$-CdSe System and the Crystal Structure of the $\mathrm{Tl}_{2} \mathrm{CdSnSe}_{4}$ compound. J. Phase Equilib. Diffus, 40, 6, 797-801.

26. Mozolyuk, M.Yu., Piskach, L.V., Fedorchuk, A.O., Parasyuk, O.V., Khyzhun, O.Y. (2017). The Tl $\mathrm{S}-\mathrm{PbS}-$ $\mathrm{SiS}_{2}$ system and the crystal and electronic structure of quaternary chalcogenide $\mathrm{Tl}_{2} \mathrm{PbSiS}_{4}$. Mat. Chem. Phys, 195, 132-142.

27. Tsisar, O., Piskach, L., Babizhetskyi, V., Levytskyi, V., Kotur, B., Marushko, L., Olekseyuk, I., Parasyuk, O. (2018). Fazovi rivnovahy v systemi $\mathrm{Tl}_{2} \mathrm{Se}_{-} \mathrm{In}_{2} \mathrm{Se}_{3}-\mathrm{GeSe}_{2}$ pry $520 \mathrm{~K}$. [Phase equilibria in the system Tl2Se $-\mathrm{In} 2 \mathrm{Se} 3-\mathrm{GeSe} 2$ at 520 K]. Visn. Lviv. u-tu. Ser. khimichna. - Bulletin of Lviv University. Chemistry series, 59(10), 46-52 [in Russian].

28. Davydyuk, G.E., Piasecki, M., Parasyuk, O.V., Myronchuk, G.L., Fedorchuk, A.O., Danylchuk, S.P., Piskach, L.V., Mozolyuk, M.Yu., AlZayed N. 2013. Opt. Mater, 35(12), 2514-2518.

29. Khyzhun, O.Y., Fedorchuk, A.O., Kityk, I.V., Piasecki, M., Mozolyuk, M.Yu., Piskach, L.V., Parasyuk, O.V., ElNaggar, A.M., Albassam, A.A., Karasinski, P. (2018). Electronic structure and laser induced piezoelectricity of a new quaternary compound TIInGe $\mathrm{S}_{8}$. Mat. Chem. Phys, 204, 336-344.

30. Myronchuk, G.L., Zamurueva, O.V., Parasyuk, O.V., Piskach, L.V., Fedorchuk, A.O., AlZayed, N.S., El-Naggar, A.M., Ebothe, J., Lis, M., Kityk, I.V. (2018). Structural and optical properties of novel optoelectronic $\mathrm{Tl}_{1-x} \mathrm{In}_{1-x} \mathrm{Si}_{x} \mathrm{Se}_{2}$ single crystals. J. Mat. Sci.: Mat. in Electr, 25(7), 3226-3232.

31. Myronchuk, G.L., Davydyuk, G.E., Parasyuk, O.V., Khyzhun, O.Y., Andrievski, R.A., Fedorchuk, A.O., Danylchuk, S.P., Piskach, L.V., Mozolyuk, M.Y. (2013). $\mathrm{Tl}_{1-\mathrm{x}} \mathrm{In}_{1-\mathrm{x}} \mathrm{Sn}_{\mathrm{x}} \mathrm{Se}_{2}(x=0,0.1,0.2,0.25)$ single-crystalline alloys as promising non-linear optical materials. J. Mat. Sci.: Mat. in Electr, 24(9), 3555-3563.

32. Abrykosov, N.Kh., Bankyna, V.F., Poretskaia, L.V. [y dr.]. (1975). Poluprovodnykovye khalkohenydy y splavy na ykh osnove. [Semiconductor chalcogenides and alloys based on them]. Moscow: Nauka, 219. [in Russian].

33. Abrykosov, N.Kh., Shelymova, N.Kh. (1975). Poluprovodnykovye materyaly na osnove soedynenyi AIVBVI [Semiconductor materials based on AIVBVI compounds] Moscow: Nauka. [in Russian].

34. Glukh, A.S., Sabov, M.Yu., Barchii, I.E., Tsigika, V.V., Sidei V.I. (2009). Formation of ternary compounds in the $\mathrm{Tl}_{2} \mathrm{Se}-\mathrm{GeSe}{ }_{2}$ system. Inorgan. Mater, 45, P, 1172-1176.

35. Houenou, P., Eholie, R. (1976). Etude du systeme $\mathrm{SnSe}_{2}-\mathrm{Tl}_{2} \mathrm{Se}$. Acad. Sci. Paris, 283, 16, 731-733.

36. Lazarev, V.B., Peresh, E.Yu., Starosta, V.Y., Mudryi, V.V. (1985). Fazovye ravnovesyia y svoistva soedynenyi v systemakh $\mathrm{Tl}_{2} \mathrm{~S}(\mathrm{Se})-\mathrm{SnS}_{2}\left(\mathrm{Se}_{2}\right)$. [Phase equilibria and properties of compounds in $\mathrm{T} 12 \mathrm{~S}(\mathrm{Se})-\mathrm{SnS} 2$ (Se2) systems]. Zhurn. neorh. Khymiy - Journal of Inorganic Chemistry, 30(6), 1502-1506 [in Russian].

37. Mucha, I., Wiglusz, K., Sztuba, Z., Gaweł, W. (2009). Solid-liquid equilibria in the quasi-binary thallium(I) selenide-tin(IV) selenide system. Сотр. Coupl. Phase Diagr. and Т И.Н. hermochem, 33, 545-549.

38. Odyn, V.V., Hrynko, V.V., Novoselova, A.V. (1986). P-T-X fazovaia dyahramma systemy CdSe-GeSe. [P-T-X phase diagram of the CdSe - GeSe system]. Zhurn. neorh. Khimiy - Journal of Inorganic Chemistry, 31(5), 1274-1277 [in Russian]. 
39. Stasova, M.M., Vainshtein, B.K. (1958). Elektronohrafycheskoe opredelenie struktury $\mathrm{Tl}_{2} \mathrm{Se}$. [Electron diffraction determination of the T12Se structure]. Krystallohrafiya-Crystallography, 3(2), 141-147 [in Russian].

40. Dittmar, G., Schafer, H. (1976). Die Kristallstruktur von germanium diselenid. Acta Cryst. B, 32, 2726-2728.

41. Busch, G., Frohlich, C., Hulliger, F., Steimeier, E. Structur, elektrische und thermoelektrische Eigenschaften von $\mathrm{SnSe}_{2}$. Helv. Phys. Acta. 34. P. 359-368.

42. Glukh O. S., Sabov M. Yu., Barchij I. E., Pavlyuk V. V., Marciniak B. (1961). Crystal structure of the $\mathrm{Tl}_{4} \mathrm{GeSe}_{4}$ ternary compound. Chem. Met. Alloys, 2009 (2), 10-14.

43. Eulenberger, G. (1982). Ternäre Thalliumchalkogenide mit $\mathrm{Tl}_{4} \mathrm{Ge}_{2} \mathrm{~S}_{6}$-Struktur. Monatsh. Chem, 113, 859-867.

44. Eulenberger, G. (1981). $\mathrm{Tl}_{4} \mathrm{Ge}_{4} \mathrm{Se}_{10}$, ein Thallium(1)selenogermanat mit adamantananalogem Anion $\left[\mathrm{Ge}_{4} \mathrm{Se}_{10}\right]^{4} /$ $\mathrm{Tl}_{4} \mathrm{Ge}_{4} \mathrm{Se}_{10}$, a Thallium(I) Selenogermanate with the Adamantane-Like Anion $\left[\mathrm{Ge}_{4} \mathrm{Se}_{10}\right]^{4}$. Z. Naturforsch, 36, 521-523.

45. Akinocho, G., Houenou, P., Oyetola, S., Eholie, R., Jumas, J.C., Olivier-Fourcade, J., Maurin, M. (1991). Étude structurale de $\mathrm{Tl}_{4} \mathrm{SnSe}_{4}$.J. Solid State Chem, 93(2), 336-340.

46. Jaulmes, S., Houenou, P. (1980). Structure cristalline du seleniure d'etain(IV) et de thallium(I): $\mathrm{Tl}_{2} \mathrm{SnSe}_{3}$. Mater. Res. Bull, 15(7), 911-915.

47. Henao, J.A., Delgado, J.M., Quintero, M. (1998). X-ray powder diffraction data and structural study of $\mathrm{Fe}_{2} \mathrm{GeSe}_{4}$. Powder Diffr, 13(4), 202-209.

48. Akselrud, L.G., Zavalii, P.Yu., Grin, Yu. et al., J. (2014). WinCSD: software package for crystallographic calculations (Version 4), 47, 803-805.

49. Ketelaar, J.A., t'Hart, W.H., Moerel, M., Polder D. (1939). The Crystal Structure of TISe, Thallous Thallic or Thallosic Selenide. Z. Kristallog, A, 101, 396-404.

50. D. Müller, G. Eulenberger, H. Hahn (1973) Über ternäre Thalliumchalkogenide mit Thalliumselenidstruktur. Z. Anorg. Allg. Chem, 398, 207-220. 